\title{
Effect of Hormone Therapy on Long-term Outcomes of Patients with Human Epidermal Growth Factor Receptor 2- and Hormone Receptor-Positive Metastatic Breast Cancer: Real World Experience in China
}

\author{
Feng Du ${ }^{\star}$, Peng Yuan ${ }^{\star}$, Jia-Yu Wang, Fei Ma, Ying Fan, Yang Luo, Bing-He Xu*
}

\begin{abstract}
Background: Among human epidermal growth factor receptor 2 (HER2)-positive breast cancer, more than half are also hormone receptor (HR)-positive. Although HR is a predictive factor for the efficacy of hormone therapy, there are still some uncertainties in regard to the effects on patients with HR-positive and HER2-positive metastatic breast cancers due to the potential resistance to hormone therapy caused by co-expression of HR and HER2. There are no clinical trials directly comparing the efficacy of hormonal therapy with chemotherapy. Materials and Methods: To examine the real-world effect of hormone therapy on patients with HR-positive and HER2-positive metastatic breast cancers, a cross-sectional study of a representative sample of the Chinese population was conducted. The study included 113 patients who received first-line and second-line palliative treatment between 2005 and 2010 in the Cancer Institute and Hospital, Chinese Academy of Medical Science. The effect of hormone therapy on overall survival (OS) was studied. Results: The patients who received hormone therapy $(n=51)$ had better overall survival in contrast to those who received chemotherapy with anti-HER2 therapy $(n=62)$ in first- or second-line treatment. The difference was of borderline statistical significance $(51.8 \mathrm{~m}$ vs 31.9m, $p=0.065)$. In addition, the effect of hormone therapy did not differ significantly with other prognostic factors, including age ( $\leq 50$ years or $>50$ years), disease free survival $(\geq 2$ years or $<2$ years) and site of metastasis (visceral or bone/soft tissue). On multivariate analysis, administration of hormone therapy was associated with a trend toward a favorable prognosis $(p=0.148, \mathrm{HR}=0.693,95 \% \mathrm{CI} 0.422-1.139)$. Age more than 50 years was the sole independent harmful prognostic factor $(p<0.001$, HR=2.797, 95\% CI 1.676-4.668). Conclusions: Our data suggest that hormonel therapy may improve outcomes of the patients with ER-positive and HER2-positive metastatic breast cancer.
\end{abstract}

Keywords: Breast neoplasm - hormonal receptor - human epidermal growth factor receptor-2 chemotherapy

Asian Pac J Cancer Prev, 16 (3), 903-907

\section{Introduction}

In primary breast cancer, the status of hormonal receptor $(\mathrm{HR})$ including the estrogen receptor $(\mathrm{ER})$ and progesterone receptor (PR) has long been recognized as a prognostic factor (Char-Hong et al., 2014). It is also a predictive factor of the efficacy of hormonal therapy (Goldhirsch et al., 2009). Another fundamental factor, HER-2 is overexpressed or amplified in approximately 15\%-25\% breast cancer (Slamon et al., 1989; Mohamad et al., 2014). HER-2 status has been confirmed to be a predictive factor of the effect of anti-HER2 therapy. The patients with HER-2 positive breast cancer were usually given the combination of chemotherapy and HER-2 targeted therapy, which could significantly prolong the PFS and OS compared with those receiving chemotherapy alone (Seidman, 2001). It is noteworthy that ER and/or
PR is positive in $36 \%-53 \%$ of the patients with HER2positive breast cancer (Slamon et al., 2001; Gianni et al., 2010; Hayashi et al., 2013). Nevertheless, there are still some uncertainties regarding to the effect of hormonal therapy on the prognosis of those patients. Firstly, several laboratory studies demonstrated that there was signal cross-talk between HR and HER-2 signal pathway, which may contribute to the resistance to hormonal therapy (Kumar et al., 2002; Chung et al., 2002; Shou et al., 2004). But the results of several clinical trials were inconsistent with the finding above (Elledge et al., 1998; De Laurentiis et al., 2005). On the other hand, hormonal therapy was considered to be the preferred treatment for patients with HR-positive metastatic breast cancer regardless of HER-2 status due to the incurable nature of this disease and its improved toxicity profile. However, recently ASCO guideline suggested the combination of 
chemotherapy and HER-2 targeted therapy be the most appropriate first-line treatment in patients with HER-2 positive and ER-positive advanced breast cancer based on the literature analysis that some first-line chemotherapy trials, such as CLEOPATRA (Swain et al., 2013), did show an OS benefit for chemotherapy and HER2-targeted therapy combination, in which patients with ER-positive breast cancer were also included (Giordano SH et al., 2014) whereas OS superiority was not observed in patients receiving hormonal therapy plus anti-HER2 therapy in both clinical trials (Johnston et al 2009; Kaufman et al., 2009), which compared the combination therapy with hormonal therapy alone.

However, until now, there are no randomized clinical trials directly comparing the effect of hormonal therapy with chemotherapy in metastatic setting due to the difficulties in designing such prospective study. Therefore, it was necessary to determine the status of hormonal therapy on the treatment of these patients. We set out to describe the clinicopathologic features and real-world outcomes of the patients with HR-positive and HER2positive metastatic breast cancer who received first-line and second-line treatment in Cancer institute and hospital (CIH), Chinese Academy of Medical Sciences(CAMS) between 2005 and 2010. Specifically, we assessed the effect of hormonal therapy in metastatic setting.

\section{Materials and Methods}

\section{Patients}

All clinical data of this study was collected from the database at Cancer Hospital and Institute, Chinese Academy of Medical Sciences (CAMS). We retrospectively reviewed the data from the patients who had HR-positive/ HER2-positive invasive primary breast cancer and metastatic disease diagnosed between 2005 and 2010 . The patients with ductal carcinoma in situ or metaplastic carcinoma were excluded.

\section{Pathologic analysis}

ER or PR was considered to be positive if $\geq 1 \%$ of the cells had nuclear staining of the receptor on immunohistochemical analysis or if the status had been coded "positive" in the medical records. HR-positive disease was defined, if ER and/or PR was positive. HER2 status was evaluated by immunohistochemical analysis or fluorescence in situ hybridization. HER2 was thought to be positive, if the receptor over-expression staining scores were $3+$ on immunohistochemical analysis or the gene copy:CEP-17 ratio was greater than 2.2, which was indicated by gene amplification on fluorescence in situ hybridization.

\section{Statistical methods}

OS referred to the period from the date of diagnosis to the date of death or the last follow-up. OS rates were estimated by the Kaplan-Meier method and compared between groups using the log-rank test. Cox proportional hazards models were used to determine the association between the type of treatment and the risk of death after adjustment of disease characteristics. All statistical analyses were done by SPSS 17 software (SPSS Inc., Chicago, IL), and $p$ value less than 0.05 was considered to be statistically significant.

\section{Results}

The baseline clinicopathological features and treatment details of these cases were shown in Table 1 . The median age was 48 years (range, $24-84$ years). The median followup time was 59.9 months (range, 4.1-217.5 months). Sixty-eight patients were dead. The median disease-free survival (DFS) was 27.4 months (range, 1-164.4 months). The median OS was 44.6 months (95\%CI:31.7 to 57.7 months) from the date of diagnosis as metastatic disease to the date of the last follow-up.

Of the 113 patients, 61.9\% (70/113) had visceral metastasis, and $35.8 \%(43 / 113)$ had merely bone or soft tissue metastasis. Metastatic breast cancer was initially diagnosed in $17.7 \%(20 / 113)$. Of the 72 patients who received first-line chemotherapy, $41.7 \%(30 / 72)$ received taxane-dependent therapy, $27.8 \%$ (20/72) received both taxane and anthracycline therapy and $16.7 \%(12 / 72)$ received venorelbine-dependent therapy. Among the 51 patients who received hormonal therapy in first-line treatment or second-line treatment, 15 patients received

Table 1. Patients and their Clinicopathologic Characteristics

\begin{tabular}{|c|c|c|c|}
\hline \multicolumn{2}{|l|}{ Characteristics } & \multirow{2}{*}{$\frac{N}{70}$} & \multirow{2}{*}{$\frac{\%}{61.9}$} \\
\hline HR status & $\mathrm{ER}+/ \mathrm{PR}+$ & & \\
\hline & $\mathrm{ER}+/ \mathrm{PR}-$ & 24 & 21.2 \\
\hline & ER-/PR+ & 19 & 16.9 \\
\hline \multirow[t]{5}{*}{$\mathrm{T}$ stage } & $\mathrm{T} 1$ & 20 & 17.7 \\
\hline & $\mathrm{T} 2$ & 36 & 31.9 \\
\hline & $\mathrm{T} 3$ & 1 & 0.9 \\
\hline & T4 & 3 & 2.7 \\
\hline & $\mathrm{N} / \mathrm{A}$ & 53 & 46.8 \\
\hline \multirow[t]{5}{*}{$\mathrm{N}$ stage } & NO & 36 & 31.9 \\
\hline & N1 & 23 & 20.4 \\
\hline & $\mathrm{N} 2$ & 19 & 16.8 \\
\hline & N3 & 13 & 11.5 \\
\hline & $\mathrm{N} / \mathrm{A}$ & 22 & 19.7 \\
\hline \multirow[t]{4}{*}{ Site of metastasis } & Bone/soft tissue & 43 & 38.1 \\
\hline & liver & 41 & 36.3 \\
\hline & Lung & 34 & 30.1 \\
\hline & Brain & 8 & 7.1 \\
\hline \multicolumn{4}{|l|}{ Adjuvant treatment } \\
\hline \multicolumn{2}{|c|}{ Adjuvant chemotherapy } & 80 & 70.8 \\
\hline \multicolumn{2}{|c|}{ Adjuvant hormonal therapy } & 65 & 57.5 \\
\hline \multicolumn{2}{|c|}{ Adjuvant anti-HER2 therapy } & 5 & 4.4 \\
\hline \multicolumn{4}{|c|}{ First-line treatment } \\
\hline \multicolumn{2}{|c|}{ Chemotherapy alone } & 72 & 63.7 \\
\hline \multicolumn{2}{|c|}{ Chemo plus anti-HER2 therapy } & 27 & 23.9 \\
\hline \multicolumn{2}{|c|}{ Hormonal therapy } & 14 & 12.4 \\
\hline \multicolumn{4}{|c|}{ Second-line treatment } \\
\hline \multicolumn{2}{|c|}{ Chemotherapy alone } & 46 & 47.4 \\
\hline \multicolumn{2}{|c|}{ Chemotherapy plus anti-HER2 therapy } & 14 & 14.4 \\
\hline \multicolumn{2}{|c|}{ Hormonal therapy alone } & 31 & 32 \\
\hline \multicolumn{2}{|c|}{ Hormonal therapy plus anti-HER2 therapy } & 6 & 6.2 \\
\hline \multicolumn{4}{|c|}{ Survival status at last followup } \\
\hline Alive & & 45 & 39.8 \\
\hline Dead & & 68 & 60.2 \\
\hline
\end{tabular}

$\overline{* H R \text {, hormonal receptor; ER, estrogen receptor; PR, progesterone }}$ receptor 
tamoxifen and 36 patients received AIs. Of the 40 patients who received anti-HER-2 therapy, 90\% (36/40) administrated trastuzumab in first-line or second-line treatment and 10\% (4/40) received lapatinib in secondline treatment.

Assessment of the effect of hormonal therapy in first-line or second-line therapy

Among the 113 patients, 51 patients received hormonal therapy in first-line or second-line therapy and 62 patients received regimen containing chemotherapy with antiHER2 therapy or not. We found a trend toward better OS in the patients who received hormonal therapy compared to those who did not (51.8m vs 31.9m, $p=0.065)$ (Figure 1).

Of the 51 patients receiving hormonal therapy, the proportion of cases who received third-line, fourth-line or fifth-line treatment was $60.1 \%, 37.3 \%$ and $21.6 \%$, respectively. In contrast, the proportion was $56.5 \%, 37.1 \%$

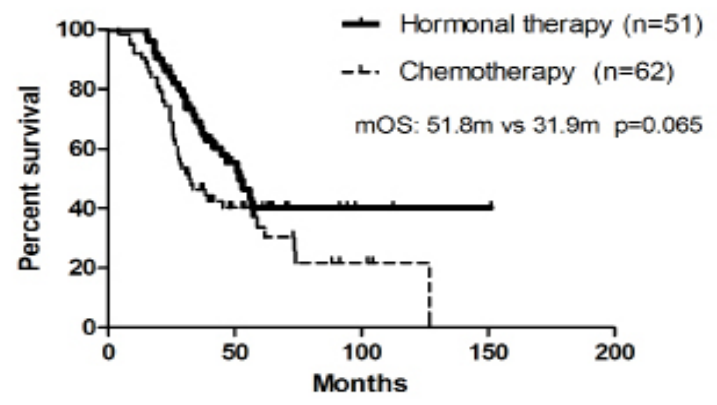

Figure 1. Comparison of the Effects of Hormonal Therapy and Chemotherapy

Table 2. The Log-rank Analysis of OS in Patients who Received Hormonal Therapy or not Stratified by Age, Metastatic Site and DFS

\begin{tabular}{lccccc}
\hline & $\begin{array}{c}\text { Hormonal therapy } \\
\mathrm{n}\end{array}$ & $\begin{array}{c}\text { No hormonal therapy } \\
\text { mOS }\end{array}$ & $\mathrm{n}$ & $\begin{array}{c}\text { mOS } \\
\text { (month) }\end{array}$ & $p$ \\
& & & & & \\
\hline Age & 31 & 56.6 & 33 & 73.6 & 0.635 \\
$\quad \leq 50$ years & 20 & 34.4 & 29 & 25.8 & 0.124 \\
$\quad>50$ years & & & & & \\
$\begin{array}{l}\text { Metastatic site } \\
\quad \text { Visceral }\end{array}$ & 30 & 50.6 & 40 & 27.6 & 0.079 \\
$\quad$ Bone/soft tissue & 21 & $\mathrm{NR}$ & 22 & 39.4 & 0.404 \\
$\begin{array}{l}\text { DFS } \\
\quad 2 \text { years }\end{array}$ & 21 & 50.8 & 26 & $\mathrm{NR}$ & 0.998 \\
$\quad<2$ years & 22 & 56.6 & 24 & 27.6 & 0.062 \\
\hline
\end{tabular}

DFS, disease-free survival; NR, not reached and $24.2 \%$ among the 62 patients without hormonal therapy, respectively.

Then, the two groups of patients were stratified by the potential prognostic factors including age $(\leq 50$ or $>50)$, sites of metastasis (visceral or bone/soft tissue) and DFS ( $\geq 2$ years or $<2$ years), and analyzed. The results were summarized in Table 2. There was no significant difference in terms of OS between each subgroups.

Compare of the effect of hormonal therapy and chemotherapy in first-line treatment

Among the 113 patients who received first-line treatment, 14 patients received hormonal therapy, and 99 patients received chemotherapy among which 72 cases accepted chemotherapy alone and 27 patients received both chemotherapy and anti-HER2 therapy. It was demonstrated that the patients who received hormonal therapy had longer overall survival than the chemotherapy arm, but the difference was not significant. (not reached (hormonal) vs $41.4 \mathrm{~m}$ (chemo), $p=0.325$ )

\section{Compare of effect of hormonal therapy and chemotherapy in second-line treatment}

Of the 97 patients who received second-line treatment, 37 patients received hormonal therapy, of which 31 patients $(83.8 \%)$ taken hormonal therapy alone and 6 patients $(16.2 \%)$ received hormonal therapy plus antiHER2 therapy, and sixty patients received chemotherapy among which 14 cases (23.3\%) taken chemotherapy plus HER 2 targeted therapy and 46 cases $(76.7 \%)$ received chemotherapy alone. As a result, better overall survival was observed in the patients who received hormonal therapy but the difference was not statistically significant(51.8 (hormonal) vs $38.4 \mathrm{~m}$ (chemo), $p=0.356$ ).

Assessment of the effect of anti-HER2 therapy in first-line or second-line therapy

Of the 113 patients, 40 patients received anti-HER2 therapy and 73 patients did not. The superiority in overall survival was observed in the patients who received antiHER2 therapy. However, the difference was not significant (56.6m vs 37.0m, $p=0.319$ ).

Univariate and multivariate analysis of prognostic factors

We performed univariate and multivariate analysis of the possible prognostic factors on the 113 patients, including treatment and clinicopathological factors. In the

Table 3. Predictors of OS in Univariate and Multivariate Cox Regression Analysis

\begin{tabular}{|c|c|c|c|c|c|c|c|c|}
\hline & \multicolumn{2}{|c|}{ Univariant analysis } & \multicolumn{4}{|c|}{ 95\%CI Multivariant analysis } & \multicolumn{2}{|c|}{$95 \% \mathrm{CI}$} \\
\hline & $p$ & HR & lower & upper & $p$ & HR & lower & upper \\
\hline age $)(\leq 50 v s>50)$ & $<0.001$ & 3.109 & 1.889 & 5.116 & $<0.001$ & 2.797 & 1.676 & 4.668 \\
\hline Site of metastasis (viseral vs bone/ soft tissue) & 0.033 & 0.559 & 0.328 & 0.954 & 0.239 & 0.718 & 0.414 & 1.246 \\
\hline Adjuvant hormonal therapy (yes $v s$ no) & 0.394 & 1.05 & 0.938 & 1.176 & & & & \\
\hline (Adjuvant HER2 targeted therapy (yes $v s$ no) & 0.126 & 1.094 & 0.975 & 1.226 & & & & \\
\hline Hormonal therapy in 1 st- or 2 nd-line treatment (no $v s$ yes) & 0.067 & 0.631 & 0.386 & 1.034 & 0.148 & 0.693 & 0.422 & 1.139 \\
\hline HER 2 targeted therapy in 1 st- or 2 nd-line treatment(no $v s$ ye) & 0.321 & 0.767 & 0.454 & 1.295 & & & & \\
\hline Chemotherapy in 1 st-or 2 nd-line treatment (no $v s$ yes) & 0.128 & 1.493 & 0.891 & 2.504 & & & & \\
\hline Third-line treatment (yes $v s$ no) & 0.161 & 0.705 & 0.432 & 1.15 & & & & \\
\hline Fourth-line treatment (yes vs no) & 0.333 & 0.78 & 0.473 & 1.289 & & & & \\
\hline Fifth-line treatment (yes $v s$ no) & 0.286 & 0.718 & 0.391 & 1.319 & & & & \\
\hline
\end{tabular}


univariate analysis, we found that age more than 50 years $(p<0.001, \mathrm{HR}=3.074,95 \% \mathrm{CI} 1.902-4.97)$ and presence of visceral metastasis $(p=0.032, \mathrm{HR}=0.571,95 \% \mathrm{CI} 0.342-$ 0.952 ) were associated with increased risk of death. On the contrary, the use of hormonal therapy in first-line or second-line treatment showed a trend toward reduced risk of death $(p=0.067, \mathrm{HR}=0.631,95 \% \mathrm{CI} 0.386-1.034)$

Similarly, in the multivariate analysis, the use of hormonal therapy showed a trend toward reduced risk of death $(p=0.148, \mathrm{HR}=0.693,95 \% \mathrm{CI} 0.422-1.139)$. Age (more than 50 years) was the sole independent harmful prognostic factor in terms of OS $(p<0.001, \mathrm{HR}=2.797$, 95\%CI 1.676-4.668.) (Table 3).

\section{Discussion}

Our results indicated that hormonal therapy may provide greater survival benefits to the patients with HRpositive and HER2-positive breast cancer than cytotoxic agents in metastatic setting. In clinical practice, hormonal therapy and chemotherapy were frequently considered for the patients with HR-positive and HER2-positive metastatic breast cancer. In metastatic setting, hormonal therapy was considered to be the preferred choice for the patients with HR-positive breast cancer in the absence of symptomatic visceral metastasis due to the improved toxicity profiles, higher quality of life and less expense compared to chemotherapy. But we still need to confirm its effect in this specific group of patients because of the more aggressive nature of HER-2 positive disease and the greater chance of developing resistance to hormonal therapy which was caused by the co-expression of HER2 and HR. Our data suggested that hormonal therapy was appropriate for the patients with HR-positive and HER-2 positive metastatic breast cancer.

It was also indicated that that any cross-talk that occurred between ER and HER2 was insufficient to degrade the effect of hormonal therapy to the patients with ER-positive and HER2-positive breast cancer. It has been asserted that the cross-talk between ER and HER2 is bidirectional and may cause resistance to tamoxifen and AIs. Dowssett et al. (Dowsett et al., 2006) found no survival benefits of tamoxifen addition in the adjuvant treatment settings in the patients with HR-positive and HER-2-positive breast cancer. However, as they noted, the sample size was relatively small $(n=75)$ and duration of tamoxifen administration was only 2 years. Furthermore, that study used a unique definition of HR status (H-score), making it difficult to compare with others. In 2005, a meta analysis showed a higher risk of disease-progression within 6 months in the patients who had HER-2-positive metastatic breast cancer and received hormonal therapy compared to those with HER-2-negative disease (HR=1.42, 95\%CI 1.32-1.52, $p<0.001)$. Nevertheless, some studies in the meta analysis used nonstandard methods like PCR, Southern-blot or ELISA to determine HER-2 status (De Laurentiis et al., 2005). Therefore, our results provided a different perspective in this area.

In contrast, in the study of Elledge et al. (Elledge RM et al., 1998), there was no significant difference in the efficacy of tamoxifen between the patients with HR positive and HER-2 positive metastatic breast cancer, including objective response rate, PFS and OS. Also, not in a metastatic setting but a adjuvant setting, Naoki Hayashi et al. (2013) found significant improvement of DFS in 128 patients with ER-positive and HER-2 positive breast cancer who received hormonal therapy after chemotherapy and trastuzumab compared to 46 patients who merely received chemotherapy and trastuzumab and a similar trend toward overall survival. Taken these findings together, it was suggested that hormonal therapy was beneficial regardless of the extent of disease.

There were also some limitations in the study. First, the sample size was relatively small, making it difficult to determine the probable subgroup of patients who may obtain greater benefits from hormonal therapy. Second, the association between quantitative expression levels of ER or PR and prognosis was not assessed, since the quantitative expression levels of some patients in the study were not available in the medical records.

In summary, our findings demonstrated that hormonal therapy may improve outcomes of the patients with ERpositive and HER2-positive metastatic breast cancer, as was significant in directing clinical practice.

\section{Acknowledgements}

All authors were involved in data acquisition or data analysis/interpretation, drafting of the manuscript and/ or its critical review, and all read and approved the final version for submission. No funding was provided for this work. Ethical approval was not required.

\section{References}

Chung YL, SheuML, YangSC, et al (2002). Resistance to tamoxifen-induced apoptosis is associated with direct interaction between Her2/neu and cell membrane estrogen receptor in breast cancer. Int J Cancer, 97, 306-12.

$\mathrm{Ng} \mathrm{CH}$, Pathy NB, Taib NA, et al (2014). Do clinical features and survival of single hormone receptor positive breast cancers differ from double hormone receptor positive breast cancers? Asian Pac J Cancer Prev, 15, 7959-64.

De Laurentiis M, Arpino G, Massarelli E, et al (2005). A metaanalysis on the interaction between HER-2 expression and response to endocrine treatment in advanced breast cancer. Clin Cancer Res, 11, 4741-8.

Dowsett M, Houghton J, Iden C, et al (2006). Benefit from adjuvant tamoxifen therapy in primary breast cancer patients according oestrogen receptor, progesterone receptor, EGF receptor and HER2 status. Ann Oncol, 17, 818-26.

Elledge RM, Green S, Ciocca D, et al (1998). HER-2 expression and response to tamoxifen in estrogen receptor-positive breast cancer: a southwest oncology group study. Clin Cancer Res, 4, 7-12.

Gianni L, Eiermann W, Semiglazov V, et al (2010). Neoadjuvant chemotherapy with trastuzumab followed by adjuvant trastuzumab versus neoadjuvant chemotherapy alone, in patients with HER2-positive locally advanced breast cancer (the NOAH trial): a randomised controlled superiority trial with a parallel HER2-negative cohort. Lancet, 375, 377-84.

Giordano SH, Temin S, Kirshner JJ, et al (2014). Systemic therapy for patients with advanced human epidermal growth factor receptor 2-positive breast cancer: american society of clinical oncology clinical practice guideline. J Clin Oncol, 
Goldhirsch A, Ingle JN, Gelber RD, et al (2009). Thresholds for therapies: highlights of the St Gallen International Expert Consensus on the primary therapy of early breast cancer 2009. Ann Oncol, 20, 1319-29.

Hayashi N, Niikura N, Yamauchi H, et al (2013). Adding hormonal therapy to chemotherapy and trastuzumab improves prognosis in patients with hormone receptorpositive and human epidermal growth factor receptor 2-positive primary breast cancer. Breast Cancer Res Treat, 137, 523-31.

Johnston S, Pippen J Jr, Pivot X, et al (2009). Lapatinib combined with letrozole versus letrozole and placebo as first-line therapy for postmenopausal hormone receptor-positive metastatic breast cancer. J Clin Oncol, 27, 5538-46.

Kumar R, Wang RA, Mazumdar A, et al (2002). A naturally occurring MTA1 variant sequesters oestrogen receptor-alpha in the cytoplasm. Nature, 418, 654-7.

Kaufman B, Mackey JR, Clemens MR, et al (2009). Trastuzumab plus anastrozole versus anastrozole alone for the treatment of postmenopausal women with human epidermal growth factor receptor 2-positive, hormone receptor-positive metastatic breast cancer: results from the randomized phase III TAnDEM study. J Clin Oncol, 27, 5529-37.

Khabaz MN (2014). Immunohistochemistry subtypes (ER/PR/ HER) of breast cancer: where do we stand in the west of Saudi Arabia? Asian Pac J Cancer Prev, 15, 8395-8400

Seidman AD, Fornier MN, Esteva FJ, et al (2001). Weekly trastuzumab and paclitaxel therapy for metastatic breast cancer with analysis of efficacy by HER2 immunophenotype and gene amplification. J Clin Oncol, 19, 2587-95.

Shou J, Massarweh S, Osborne CK, et al (2004). Mechanisms of tamoxifen resistance: increased estrogen receptor-HER2/ neu cross-talk in ER/HER2-positive breast cancer. J Natl Cancer Inst. 96, 926-35.

Slamon DJ, Godolphin W, Jones LA, et al (1989). Studies of the HER-2/neu proto-oncogene in human breast and ovarian cancer. Science. 244, 707-12.

Slamon DJ, Leyland-Jones B, Shak S, et al (2001). Use of chemotherapy plus a monoclonal antibody against HER2 for metastatic breast cancer that overexpresses HER2. $N$ Engl J Med. 344, 783-92.

Swain SM, Kim SB, Cortes J, et al (2013). Pertuzumab, trastuzumab, and docetaxel for HER2-positive metastatic breast cancer (CLEOPATRA study): overall survival results from a randomised, double-blind, placebo-controlled, phase 3 study. Lancet Oncol, 14, 461-71. 